\title{
Functional self-similarity and renormalization group symmetry in mathematical physics
}

\author{
Vladimir F. Kovalev* and Dmitrij V. Shirkov ${ }^{\dagger}$
}

\begin{abstract}
The results from developing and applying the notions of functional self-similarity and the Bogoliubov renormalization group to boundary-value problems in mathematical physics during the last decade are reviewed. The main achievement is the regular algorithm for finding renormalization group-type symmetries using the contemporary theory of Lie groups of transformations.
\end{abstract}

\section{Introduction}

The notion of functional self-similarity (FS) was introduced in mathematical physics by one of the authors in the early 1980s [1] (see also [2, 30). The basis of this introduction is that solutions of a wide class of problems analyzed by the renormalization group ( $R G$ ) method are invariant w.r.t. the group transformations that involve not only natural independent variables of a problem but also parameters of boundary conditions imposed at some "reference" point. The RG transformation then corresponds to reparameterizing a solution by changing (shifting or rescaling) an independent (coordinate) variable while simultaneously performing a functional transformation of (boundary) characteristics of the selected functions when passing to another reference point. The corresponding "transformation functions" are governed by group functional equations.

More precisely, we consider the so-called renormalization transformations (the Dyson transformations) in quantum field theory (QFT), which constitute a continuous oneparameter group, i.e., the Lie group of transformations (if we use a standard mathematical language). This group was discovered [4] and used [5] to analyze QFT singularities. We call this group the QFT RG or the Bogoliubov RG to distinguish it from the approximate RG, which was introduced by Wilson [6] to analyze critical phenomena in statistical physics problems.

\footnotetext{
*Institute for Mathematical Modeling, RAS, Moscow, Russia, e-mail: kovalev@imamod.ru

${ }^{\dagger}$ Bogoliubov Laboratory for Theoretical Physics, Joint Institute for Nuclear Research, Dubna, Russia, e-mail: shirkovd@thsun1.jinr.ru
} 
The reasoning above pertains to the $\mathrm{RG}$ transformations considered within the QFT approach. In the middle 1980s, the RG method became widely applied to classical problems in mathematical physics; this was initially connected with transforming and applying methods developed for QFT and statistical physics. Such applications were based, first, on the fact that physical problems manifest the FS property, which permits segregating the characteristic variables of a problem (independent and dynamic variables, parameters, and boundary data) and finding transformations that preserve the solution, and, second, on an advantageous mathematical description that uses only differential and integraldifferential equations to describe the model. The RG approach is also favorable because the corresponding RG-type transformations can be constructed using regular methods, which are used to find symmetries in the group analysis of differential equations (DE). Substantial progress has been achieved in group analysis since the RG method first appeared in theoretical physics several decades ago.

The transition to boundary-value problems in mathematical physics enriched the mathematical content while preserving the main property of the FS, i.e., the solution of a physical problem is invariant w.r.t. a special class of transformations. The initial notion of the FS transformations as point transformations of independent variables was recently generalized to contact transformations, transformations defined by formal series, etc. Because the infinitesimal transformation approach is convenient for the group analysis of mathematical models based on DEs, we can also formulate the FS in terms of infinitesimal operators determining the corresponding RG symmetry (RGS). The operators then appear as a result of the standard RGS construction procedure, and the FS condition arises in the course of this procedure.

We review the evolution of the FS notion connected with the implementation of the RGS method in mathematical physics and describe the results obtained in the RGS framework. In Sec. 2, we introduce both infinitesimal and finite RG transformations, establish their connection with the mathematical physics notion of powerlike self-similarity (automodelness), and introduce the notion of the FS. We then construct RGSs in mathematical physics. The FS property appears as an ingredient of this construction, and we trace how the FS notion changes when passing from the Bogoliubov RG to mathematical physics models.

In Sec. 3, we consider examples of the FS transformations that are close to the QFT transformations because they are realized as groups of one-parameter transformations w.r.t. some independent variables. We present examples where the group extends and generates FS transformations with operators constituting a finite-dimensional algebra. Because an ODE or a system of such equations governing the mathematical model discussed in this section coincides with the Lie equations arising in the QFT applications of the RG method, the formalism developed for ODEs can also be applied to Lie equations.

In Sec. 4, we collect examples of FS conditions written either as a first-order PDE or as higher-order differential relations. We discuss a novel form of the FS transformations that can be presented as formal infinite series, not as traditional algebraic relations. The FS 
transformations acquire such a form when the RGS are of the Lie-Bäcklund group type, and the FS condition claims that the particular solution of a boundary-value problem is invariant w.r.t. these transformations.

In Sec. 5, we analyze the FS conditions for systems with small parameters. The FS conditions in such systems permit finding approximate RGSs and using them to solve problems with arbitrary boundary conditions. We present examples of approximate RGSs for several problems in nonlinear physics.

In Sec. 6, we describe the perspectives of the FS and its use to construct RGSs in a broad class of mathematical physics problems.

\section{The QFT RG and the FS condition}

\subsection{Simple Bogoliubov RG transformations}

To illustrate the FS notion, we consider the simplest RG transformation, which is a simultaneous one-parameter transformation of one independent variable in a problem (the coordinate $\ell$, for example) and a characteristic $g$ of a solution,

$$
T(\lambda):\left\{\ell \rightarrow \ell^{\prime}=\ell-\lambda, g \rightarrow g^{\prime}=G(\lambda, g)\right\} \quad, \quad G(0, g)=g .
$$

The function $G(\ell, g)$ satisfies the functional relation

$$
G(\ell+\lambda, g)=G(\ell, G(\lambda, g))
$$

which corresponds to the group composition law for the transformation operators, $T\left(\lambda_{1}\right)$. $T\left(\lambda_{2}\right)=T\left(\lambda_{1}+\lambda_{2}\right)$.

Transformation operator (1) can sometimes be conveniently represented explicitly by writing a transformation of a function $F(\ell, g)$ in the form

$$
T(\lambda) F(\ell, g)=e^{-\lambda R} F(\ell, g)
$$

using the infinitesimal RGS operator (or the RG operator)

$$
R=\partial_{\ell}-\beta(g) \partial_{g}, \quad \beta(g)=\left.\frac{\partial G(\lambda ; g)}{\partial \lambda}\right|_{\lambda=0},
$$

whose coordinate $\beta(g)$ is the derivative of the function $G$ at $\lambda=0$. The infinitesimal RG transformations can be conveniently represented via the operator $R$; the group nature of the operator $T(\lambda)=e^{-\lambda R}$ is obvious from "finite shift" representation (3).

The condition

$$
R I(\ell, g) \equiv \partial_{\ell} I-\beta(g) \partial_{g} I=0,
$$

\footnotetext{
${ }^{1}$ The set of group variables of the generalized Lie group of transformations (the Lie-Bäcklund group) includes derivatives of the desired functions w.r.t. independent variables.
} 
determines an invariant of the RG transformation, which is the function $I(\ell, g)=\tilde{I}(G(\ell, g))$ $\equiv I(0, G(\ell, g))$ of one argument in this case because the transformation function $G(\ell, g)$ is an invariant itself by virtue of Eq. (2). The condition

$$
R C(\ell, g)=\varphi(g) C(\ell, g)
$$

determines a covariant, i.e., a quantity that transforms according to a representation of the FS group or the RG. Covariants are important for QFT applications of the RG.

The representation of a RG transformation via an infinitesimal operator $R$ is equivalent to finite transformation (3). Functional equation (2) follows from the characteristic equation for the operator $R$, and for a given function $\beta$, an explicit expression for $G$ can be constructed by solving the corresponding Lie equations for operator (4),

$$
-d \ell^{\prime}=\frac{d g^{\prime}}{\beta\left(g^{\prime}\right)}=d \lambda
$$

with the boundary conditions $\left.\quad \ell^{\prime}\right|_{\lambda=0}=\ell$ and $\left.g^{\prime}\right|_{\lambda=0}=g$.

It is important in what follows that the invariance of the function $I$ w.r.t. the $\mathrm{RG}$ transformations written as Eq. (5) is equivalent to the vanishing condition for the coordinate $\varkappa$ of operator (雨), which is written in the canonical form as

$$
\bar{R}=æ \partial_{I}, \quad æ \equiv I_{\ell}-\beta(g) I_{g}=0 .
$$

This condition must be considered on a particular solution $I(\ell, g)$ of the boundary-value problem.

The "exponentiated" variables

$$
x=e^{\ell}, \quad a=e^{\lambda}, \quad \bar{g}(a, g)=G(\lambda, g),
$$

are natural in QFT. In these variables, the group transformation $T(\lambda)=T_{a}$ becomes

$$
T_{a}:\left\{x^{\prime}=x / a, g^{\prime}=\bar{g}(a, g)\right\}, \quad \bar{g}(1, g)=g,
$$

and

$$
\bar{g}(x, g)=\bar{g}(x / a, \bar{g}(a, g)), \quad T_{a} \cdot T_{b}=T_{a b} .
$$

Functional equation (10) and transformation (9) appear, for example, in a QFT with one coupling constant in the massless limit, where the dimensionless constant $x=Q^{2} / \mu^{2}$ is the ratio of the squared transferred four-momentum $Q$ to the squared "normalized" momentum $\mu, g$ is the coupling constant, and the invariant $\bar{g}$ is the invariant (or effective) coupling function. More complicated QFT RG transformations are generalizations of transformation (9) obtained by "multiplying" solution characteristics, $g \rightarrow\{g\}=\left(g_{1}, g_{2}, \ldots, g_{k}\right)$, and by introducing additional parameters in the functions $\bar{g}_{i}(x,\{g\})$ (see Sec. 49 in [0]]). 
In the QFT model with two coupling constants $g_{1}=g$ and $g_{2}=h$ and one particle mass $m$, for example, the transformation $T_{a}$ becomes

$$
\begin{gathered}
T_{a}:\left\{x^{\prime}=x / a, \quad y^{\prime}=y / a, \quad g^{\prime}=\bar{g}(a, y ; g, h), \quad h^{\prime}=\bar{h}(a, y ; g, h)\right\}, \\
\bar{g}(1, y ; g, h)=g, \quad \bar{h}(1, y ; g, h)=h, \quad y=Q^{2} / \mu^{2} .
\end{gathered}
$$

In the case of several coupling constants, the characteristic equations that are connected with the infinitesimal operator

$$
\begin{gathered}
R=x \partial_{x}+y \partial_{y}-\beta_{1}(y ; g, h) \partial_{g}-\beta_{2}(y ; g, h) \partial_{h}, \\
\beta_{1}(y ; g, h)=\left.\frac{\partial \bar{g}(\xi, y ; g, h)}{\partial \xi}\right|_{\xi=1}, \quad \beta_{2}(y ; g, h)=\left.\frac{\partial \bar{h}(\xi, y ; g, h)}{\partial \xi}\right|_{\xi=1},
\end{gathered}
$$

become the system of first-order PDEs

$$
x \frac{\partial \bar{g}(x, y ; g, h)}{\partial x}=\beta_{1}\left(\frac{y}{x} ; \bar{g}, \bar{h}\right), \quad x \frac{\partial \bar{h}(x, y ; g, h)}{\partial x}=\beta_{2}\left(\frac{y}{x} ; \bar{g}, \bar{h}\right),
$$

however, all these one-parameter transformations are based on the transformation of a single independent argument $x$.

\subsection{Self-similarity and the FS}

In a particular case where the function $\bar{g}$ is linear in its second argument, $G \sim g$, solutions of Eq. (I0) have a powerlike dependence on the argument $x$, i.e., $\bar{g}(x, g)=g x^{k}$, where $k$ is a number, and transformations (9) become transformations of powerlike self-similarity (the so-called automodel transformations)

$$
P_{a}:\left\{x^{\prime}=x / a, \quad g^{\prime}=g a^{k}\right\}
$$

which are commonly used in problems in gas and liquid dynamics. From this standpoint, transformations (9) and (10) for arbitrary $G, \bar{g}$ are functional generalizations $g x^{k} \rightarrow \bar{g}(x, g)$ of customary self-similarity transformations and can therefore be called the functional self-similarity transformations [1]; this term is a synonym for the RG transformations.

Therefore, in the RG-transformation framework, FS reflects the group nature of functional relations. The universality of the FS formulation in the QFT and classical physics models is due to the common scaling transformation and common functional transformation of the solution characteristic $g_{\mu}=\bar{g}(\mu, g)$. Various realizations of the RG differ only in the form of the function $\beta(g)$, which is customarily calculated using an approximate solution obtained, as a rule, within the perturbation theory (PT). Therefore, the main role of the FS until recently was to establish a posteriori that a system under consideration admits functional transformations with a group structure. Group transformations themselves followed from additional considerations concerning system solutions [8]. 


\subsection{Constructing the RGS}

The situation changed substantially after passing to investigating boundary-value problems in mathematical physics. The FS property of a desired solution here results in functional relations (which follow from the group nature of a solution) or in symmetries of the RG (=FS) type in the infinitesimal form. In contemporary mathematical physics models, RGSs can be regularly found by using the scheme in [9], which naturally incorporates the FS and its formulation as the invariance condition for a particular solution of a boundary-value problem.

To demonstrate the role of FS in constructing RGSs, we recall the four main steps in this construction. The first step is to construct a special RG manifold (differential, integral-differential, etc.), which differs, generally speaking, from the manifold determined by the initial system of equations describing the physical system under investigation. The second step is to find the maximum extended transformation group $\mathcal{G}$ admitted by the $\mathrm{RG}$ manifold. The third step is to restrict the obtained group $\mathcal{G}$ on a solution (exact or approximate) of a boundary-value problem. The transformation group that appears, which is just called the RG, is a set of infinitesimal operators $R_{i}$, each of which contains a solution of the boundary-value problem in its invariant manifold. The fourth and final step is to use the infinitesimal $\mathrm{RG}$ operators $R_{i}$ to construct finite transformations of the group and to obtain an analytic expression for the solution of the boundary-value problem.

The first and principal step in this scheme for RGS construction can be realized in different ways depending on the mathematical model structure and on the type of boundary conditions [9]. The desired RG manifold can be obtained by adding parameters that enter the initial equations and boundary data to extend the list of group variables or appending to this list derivatives of the given dependent variables or nonlocal variables. We can also increase the number of initial equations by writing boundary data either in the embedding equation form or as additional differential constraints. Sometimes, the admitted group can be extended by dropping small parameters to simplify the initial equations.

The maximum group $\mathcal{G}$, which is calculated for the $\mathrm{RG}$ manifold using the modern group analysis algorithms [10 in the second step, is not yet the RG, because it is not connected with a particular solution.

The third step constructs the RG itself; namely, we restrict the obtained group $\mathcal{G}$ on a particular exact or approximate solution of the selected boundary-value problem. Mathematically, the restricting procedure implies "combining" the canonical coordinates of operators of the group admitted by the RG manifold. The vanishing condition for the sum of these coordinates on the solution of the boundary-value problem (the condition of invariance w.r.t. the RGS operator, which is analogous to condition (8))) results in a system of algebraic identities, which relate coordinates of different operators and therefore generate the desired RGSs. From the physical standpoint, the verification of this condition in each actual case is the realization of the FS principle, which therefore establishes the invariance of the particular solution w.r.t. the $\mathrm{RG}$ transformations. On the other 
hand, this step substantially relies on the PT solution of the problem under investigation simultaneously realizing the general principle of the $\mathrm{RG}$ method consisting in constructing an improved (in comparison with the PT) solution. In this scheme, the PT parameter can be any physically appropriate parameter or a set of such parameters.

Verification of the FS condition in the RGS construction scheme above differs from the procedure for verifying the invariance of the analyzed solutions w.r.t. an iterated sequence of scaling transformations, which are used to analyze the behavior of a physical system in the theory of critical phenomena [6] and are also called RG transformations although they do not constitute a group in the general case (in contrast to the QFT models, where the group property is especially proved [7, 8]). This scheme (we call it the Wilson RG) is now widely used in mathematical physics to analyze the asymptotic behavior of DE solutions [11, 12] and to construct the envelope of the solution family [13]. When applying the Wilson RG to mathematical physics problems, the algorithm for improving the PT solutions that contain singularities consists of introducing additional parameters in the solutions, using these parameters to remove singularities, and demanding the solutions to be independent of the way these parameters are introduced [11]. Whether such a construction is consistent with the transformation group of the desired boundary-value problem solution remains an open question although this algorithm works successfully in some particular cases.

The RGS in mathematical physics is defined by a set of RGS generators. Therefore, as in the classical group analysis of DEs, it suffices to consider only infinitesimal transformations that are characterized by an infinitesimal operator algebra. We note that this algebra often consists of more than one operator in contrast to the QFT-type models where we typically have only one RG operator. Both the dimension and the construction of the RG operator algebra depend on the mathematical model and on the type of boundary conditions.

The RGS group, which is restricted on a solution of a boundary-value problem, can be not only a point Lie group but also a Lie-Bäcklund group, an approximate transformation group, a nonlocal symmetry group, a non-Lie symmetry group, etc. [9]. We present the FS conditions and various forms of the FS transformations pertaining to concrete problems in which the RGS is used.

\section{The FS analysis of systems that are close to quan- tum field systems}

We begin with the FS transformations for systems described by ODEs and, for simplicity, consider a boundary-value problem for the function $u(t)$ satisfying a first-order ODE [15], 14, 9] with the parameters $b^{k}(k=1,2, \ldots)$ explicitly entering the equation:

$$
u_{t}=f\left(t, u, b^{k}\right),
$$




$$
u(\tau)=x .
$$

This example is not only methodologically important; it illustrates the FS application to QFT where such equations appear in the RG method when analyzing a system of DEs for invariant coupling functions.

Constructing the RGS for boundary-value problem (14), (15) using the algorithm in (9) consists in adding to (14) the embedding equation, which has the form of a linear first-order PDE,

$$
u_{\tau}+f\left(\tau, x, b^{k}\right) u_{x}=0 .
$$

The system of equations (14) and (16) determines the desired RG manifold in the space of all group parameters $\left\{u, t, \tau, x, b^{k}\right\}$, which are not only dependent and independent variables and the parameters of initial equation (14) but also the parameters $\tau$ and $x$ entering the boundary data.

The symmetry group $\mathcal{G}$ admitted by manifold (14), (16) depends on the form of the function $f$. In the practically important case (the ultraviolet limit of QFT models) where this function does not depend explicitly on time $t$ and only three parameters $b^{1} \equiv a$, $b^{2} \equiv b$, and $b^{3} \equiv c$ enter the initial equation, i.e., where $f=f(u, a, b, c)$, the admitted group $\mathcal{G}$ is determined by the seven-term operator

$$
X=\sum_{i=1}^{7} \alpha_{i} X_{i}
$$

The first two functions $\alpha_{1}$ and $\alpha_{2}$ in (17) are arbitrary functions of all seven group variables $\{t, \tau, x, a, b, c, u\}$, and the remaining functions depend arbitrarily on the parameters $a, b$, and $c$ and on the combinations $\tilde{t}=t-\langle 1 / f(u)\rangle$ and $\tilde{\tau}=\tau-\langle 1 / f(x)\rangle$. The angle brackets denote integrals w.r.t. the respective variable $u$ or $x$. Explicit expressions for three of the seven operators entering (17) are

$$
\begin{gathered}
X_{1}=\partial_{t}+f(u) \partial_{u}, X_{3}=f(u) \partial_{u} \\
X_{5}=f(x)<f_{a}(x) / f^{2}(x)>\partial_{x}+f(u)<f_{a}(u) / f^{2}(u)>\partial_{u}+\partial_{a} .
\end{gathered}
$$

The remaining operators are obtained by substitutions: $X_{2}$ and $X_{4}$ are obtained from $X_{1}$ and $X_{3}$ with the respective substitutions $t \rightarrow \tau$ and $u \rightarrow x$, and $X_{6}$ and $X_{7}$ from $X_{5}$ with the respective derivative substitutions $\partial_{a} \rightarrow \partial_{b}$ and $\partial_{a} \rightarrow \partial_{c}$.

The operation of restricting the group $\mathcal{G}$ is the verification of the imposed FS condition, which is analogous to the equality $\varkappa=0$ in (8), i.e., the solution must be invariant w.r.t. the $\mathrm{RG}$ transformations, or, in other words, the coordinate of the canonical operator $X$ must vanish on the solution of the initial problem $u=U(t, x, \tau, a, b, c)$. If a restriction of the group $\mathcal{G}$ admitted by manifold (14), (16) on an (approximate) solution of problem (14), (15) is fulfilled, the "restricted symmetries," which we call RGSs, I appear.

\footnotetext{
${ }^{2}$ Some $R$ operators of RGSs thus defined may result from symmetries of the equations.
} 
Which perturbation series becomes the function $U$ depends on the actual problem setting. For instance, for a polynomial function $f$,

$$
f=a u^{2}+b u^{3}+c u^{4}
$$

we can choose the PT over the variables $(t-\tau)$ or over the parameter $a, b$, or $c$. Examples of restricting the group on a solution with a PT in $a$ with $b=c=0$ was considered in [14, 9], and with a PT in $b$ with $a=1$ and $c=0$ in [9]. We present two possible RGS operators appearing after such restrictions [14, 9],

$$
\begin{gathered}
R_{1}=x^{2} \tau \partial_{x}+\partial_{a}+u^{2} t \partial_{u}, b=c=0 \\
R_{2}=\left(x^{2}(1+b x) \tau+x\right) \partial_{x}+\left(u^{2}(1+b u) t+u\right) \partial_{u}-b \partial_{b}, a=1, c=0 .
\end{gathered}
$$

We can now obtain the solution of the Cauchy problem if we write the condition for its invariance w.r.t. the RGS operator, namely, the FS condition, which is a linear first-order PDE. For instance, for the operator $R_{1}$, we have

$$
t u^{2}-x^{2} \tau u_{x}-u_{a}=0
$$

Solving the characteristic equations (the Lie equations) for this equation, we obtain the desired solution of the boundary-value problem for $b=c=0$. The FS condition of type (20) is used twice: first, when constructing the RGS operators, we substitute an approximate solution $U$ for $u$ in (20); second, when finding the solution of the boundaryvalue problem, we use these RGS operators.

In both examples, constructing the PT is easy and results either in the powerlike dependence or in a combination of powerlike, reciprocal, and logarithmic dependencies on the initial data. In this algorithm, constructing the PT in powers of $b$, which gives the second operator $R_{2}$ in (19), starts from the unperturbed state, which is chosen to be the solution of the boundary-value problem at $a=1$ obtained by applying the first operator $R_{1}$, i.e., in the approximation of strong nonlinearity (w.r.t. the parameter $a$ ). Therefore, the improvement of the PT using the operator $R_{2}$ is reduced to the consequent improvement of the PT for the boundary-value problem with the function $f=a u^{2}+b u^{3}$ first in the parameter $a$ and then in the parameter $b$ using the respective one-parameter groups $R_{1}$ and $R_{2}$.

In many cases, such a procedure of improving the PT consecutively over several parameters of a model fails. One possible reason is the absence of a "nonlinear" unperturbed solution. For the function $f$ discussed here, which is chosen as a polynomial in $u$, constructing the PT in $c$ with fixed $a$ and $b$ is not so simple as constructing the PT at $c=0$, because in the case with fixed $a$ and $b$, the unperturbed state is determined in terms of the Lambert function [16], which admits no simple analytic representation (see also [17]).

Therefore, constructing an RGS that generates group transformations w.r.t. several parameters simultaneously and improves a PT that admits an easily found unperturbed 
solution is important. In fact, we want to construct a two-parameter RG. This problem will be discussed in detail elsewhere; we consider only one example of such an RG here:

$$
\begin{aligned}
& R_{3}=-<f_{b}(u) / f^{2}(u)>\partial_{t}-<f_{b}(x) / f^{2}(x)>\partial_{\tau}+\partial_{b}, \\
& R_{4}=-<f_{c}(u) / f^{2}(u)>\partial_{t}-<f_{c}(x) / f^{2}(x)>\partial_{\tau}+\partial_{c} .
\end{aligned}
$$

Verifying the FS conditions, i.e., restricting the group $\mathcal{G}$ on the PT solution over the two parameters $b \rightarrow 0$ and $c \rightarrow 0$ simultaneously is not difficult technically, and using the finite transformations generated by the operators $R_{3}$ and $R_{4}$, which generate a two-dimensional algebra, we obtain the desired solution of problem (14), (15). Therefore, using the twoparameter $\mathrm{RG}$, we can avoid mathematical problems that arise when using one-parameter RGs.

We conclude this section with a few remarks. We have demonstrated the use of the FS condition to construct RGSs that improve the PT over the parameters $a, b$, and $c$ entering the equation, although the corresponding operators by no means exhaust an infinite set of RGSs, which is parameterized by a continuum set of the RG operator coordinates [15, 14, 9]. Quite analogously, RGS operators that improve a PT w.r.t. an appropriate combination of dynamic variables and initial data (e.g., w.r.t. the difference $t-\tau)$ can be found. Examples of such RGSs are presented in the following sections. We only mention here that the FS condition in the form of equality (8) is obviously analogous to FS condition (20) pertaining to boundary-value problem (14), (15). The RGS examples and the corresponding FS condition considered in this section follow from a single ODE. Similar constructions are valid for systems of ODEs depending on several parameters. Examples of embedding equations for such systems, which were used to find RGSs, can be found in [14].

\section{The FS conditions for PDEs}

In this section, we consider systems that are described by mathematical models based on PDEs or systems of PDEs. In contrast to the previous section, where the appearance of first-order partial derivatives in FS conditions is due to a transformation of parameters in initial equations and due to embedding equations, which imply taking the boundary conditions into account, we here demonstrate boundary-value problems for which the FS conditions are systems of DEs (constraints) that contain higher-order partial derivatives w.r.t. the independent variables. The differential formulation of the FS conditions then permits constructing the desired solution of the problem; finding finite FS transformations results in formal power series (see [18, Vol. 3, Chap. 1). In addition, we give an example of an FS condition (simpler than in Sec. 3) in the ODE form whose solution also reconstructs the solution of the boundary-value problem from the given PT. 


\subsection{Boundary-value problem for the Burgers equation}

An explicit example, which illustrates the variety of the FS condition formulations for systems based on PDEs, is provided by the boundary-value problem for the modified Burgers equation,

$$
\begin{gathered}
u_{t}-a u_{x}^{2}-\nu u_{x x}=0, \\
u(0, x)=f(x),
\end{gathered}
$$

with the nonlinearity parameter $a$ and dissipation parameter $\nu$. The continuous point symmetry group admitted by manifold (22) is defined by nine operators. Six of them are symmetries of the equation and have been discussed in the literature (see, e.g., [18, Vol. 1, Chap. 1, p. 183). They correspond to the projective transformation, the dilation transformation in the plane $(t, x)$, translations along the axes $t, x$, and $u$, and the Galilean transformations. The seventh operator is the operator of an infinite Abelian ideal $X_{\infty}=$ $\alpha \exp (-a u / \nu) \partial_{u}$ of the group; the coordinate of this ideal is parameterized by the function $\alpha(t, x, a, \nu)$ of four group variables restricted by the linear parabolic equation

$$
\alpha_{t}-\nu \alpha_{x x}=0
$$

which coincides with the linear part $(a=0)$ of initial equation $(\sqrt[22]{)}$. Eventually, when we interpret the equation parameters as independent variables, two more operators appear and involve these parameters in the group transformations; these operators correspond to scaling transformations of the respective variables $a$ and $\nu$ [19].

Restricting the group admitted by manifold (22) on the solution $u=U(t, x, a, \nu)$ of the Cauchy problem, i.e., verifying the FS condition, results in an algebraic relation, which expresses the coordinate of the infinite-dimensional subgroup generator (the function $\alpha$ ) through the coordinates of the remaining eight operators at any time $t$, including $t=0$ when this solution $U(0, x, a, \nu)=f(x)$ is known from boundary condition (23). Using the standard representation for the solution of a linear equation on the function $\alpha$ with the initial value $\alpha(0, x, a, \nu)$ obtained from the FS condition and substituting this representation in the formula that determines the general element of the Lie algebra, we obtain the desired RGS operators. Thus, we obtain the RGSs for boundary-value problem (22), (23) by combining the symmetries of the eight-dimensional algebra generated by the above operators and symmetries of the infinite-dimensional subalgebra generated by the opera-

tor $X_{\infty}$. Each of the eight RG operators obtained (and their linear combinations whose coefficients are arbitrary functions of $a$ and $\nu$ ) contains a solution of the Cauchy problem $u=U(t, x, a, \nu)$ in the invariant manifold and permits finding group transformations of both the variables $\{t, x, a, \nu\}$ and various functionals (local and nonlocal) of the solution.

We present two such RGS operators, which improve the corresponding PTs over the parameter $a$ (operator $R_{5}$ ) and the independent variable $t$ (operator $R_{6}$ ),

$$
R_{5}=\partial_{a}+\frac{1}{a}\left(-u+\exp \left(-\frac{a u}{\nu}\right)<f(x)>\right) \partial_{u}
$$




$$
R_{6}=\partial_{t}+\exp \left(-\frac{a u}{\nu}\right)<a f_{x}^{2}+\nu f_{x x}>\partial_{u}
$$

The double angle brackets here are integral convolutions of the corresponding functions with the fundamental solution $G(t, x, \nu)$ of the linear equation for the function $\alpha$ multiplied by the exponent of the function $f$ from boundary condition (23):

$$
<F(x)>\equiv \frac{1}{\sqrt{4 \pi \nu t}} \int_{-\infty}^{\infty} d y F(y) \exp \left(-\frac{(x-y)^{2}}{4 \nu t}+\frac{a f(y)}{\nu}\right) .
$$

The invariance conditions for a solution of a boundary-value problem (the FS conditions) corresponding to RG operators (24) and (25) are the two first-order ODEs

$$
\begin{aligned}
& -u_{a}-\frac{u}{a}+\frac{1}{a} \exp \left(-\frac{a u}{\nu}\right)<f(x)>=0, \\
& -u_{t}+\exp \left(-\frac{a u}{\nu}\right)<a f_{x}^{2}+\nu f_{x x}>=0 .
\end{aligned}
$$

Approximate solutions of Eq. (22) can be extended in parameters $a$ or $t$ by solving either Eq. (26) or (27) with the proper initial conditions. This eventually leads to the common exact solution $u=(\nu / a) \ln \langle\langle 1\rangle\rangle$, which is valid for all values of the parameters $a$ and $t$ [19].

In the example above, FS condition (26) for the boundary-value problem for a PDE becomes a first-order ODE. Formally, this equation is simpler than the FS conditions formulated for the ODE boundary-value problems in Sec. 3 and differs from the corresponding FS conditions for QFT models. On the other hand, formulating boundary conditions for a first-order ODE in the embedding equations language, we notice that condition (26) can be treated as an embedding equation for boundary-value problem (22), (23) with the embedding parameter $a$. In turn, the invariant embedding method can also be applied to boundary-value problem (26) with the variables $\{a, u\}$ and the initial condition $u=u_{0}(t, x, \nu)$ at $a=0$. Then, because the initial value $u_{0}$ depends on the "parameters" $\{t, x, \nu\}$ entering ODE (26), the corresponding embedding equation becomes integraldifferential [15]. The set of the RGS operators for boundary-value problem (22), (23) includes not only the above FS conditions in the form of first-order ODEs but also operators that result in FS conditions both in the form of first-order PDEs, which are analogous to the operators previously considered, and in the form of mere algebraic relations [19]. Therefore, various FS can relate to the solution of the same boundary-value problem.

\subsection{The boundary-value problem for nonlinear optic equations}

It is sometimes impossible to make a boundary-value problem PT and the FS conditions consistent if we confine ourselves to only point symmetries. Below, we consider an example where the FS condition is a second-order PDE. We now consider the following boundaryvalue problem for the system of equations for the nonlinear optics of collimated wave beams:

$$
v_{t}+v v_{x}-\alpha n_{x}=0, \quad n_{t}+v n_{x}+n v_{x}=0,
$$




$$
v(0, x)=0, \quad n(0, x)=N(x) .
$$

Here, the dimensionless coordinates $t$ and $x$ describe the spatial evolutions of the derivative $v$ of the beam eikonal and of the dimensionless intensity $n$ in the direction into the bulk and in the transverse direction, and $\alpha$ is the parameter of the nonlinear refraction. The hodograph transformation reduces (28) to the system of linear equations in the variables $\tau=n t$ and $\chi=x-v t$

$$
\begin{gathered}
\tau_{w}-n \chi_{n}=0, \quad \chi_{w}+\alpha \tau_{n}=0, \\
\tau(0, n)=0, \quad \chi(0, n)=H(n) .
\end{gathered}
$$

where we use the notation $w=v / \alpha$. Formal construction of the RGS and analysis of the FS conditions for system (30) could be as for the Burgers equation. As previously, a finitedimensional subalgebra of point symmetry operators and its infinite-dimensional ideal arise. However, the procedure of restricting this ideal in order to obtain the RGS [20, 21] implies solving the system of linear PDEs, which coincides with initial system (30); this is an intrinsic feature of linear equations.

Therefore, we construct the RGS using not the point symmetries but the Lie-Bäcklund symmetries (see [18], Vol. 3, Chap. 1), for which the terms "higher" and "generalized" symmetries are also used. The desired RGS operators for boundary-value problem (30), (31) can then be conveniently written in the canonical form

$$
R=f \partial_{\tau}+g \partial_{\chi}
$$

where the coordinates $f$ and $g$ are functions in the extended space of group variables, which, in addition to the set $(\tau, \chi, w, n, \alpha)$, includes derivatives of $\tau$ and $\chi$ of arbitrary finite order in $n$ (and, perhaps, in $\alpha$ ). The problem of constructing the Lie-Bäcklund symmetries for boundary-value problem (30), (31) was discussed in detail in [20]. It was shown that these symmetries are generated by operators of form (32) whose coordinates are linear combinations of $\tau, \chi$, and their first- and second-order derivatives depending on $w$ and $n$. We write the expressions for the coordinates of only three operators:

$$
\begin{aligned}
& f_{1}=-\tau / 2+n \tau_{n}+(1 / 2) n w \chi_{n}, \quad g_{1}=-(\alpha w / 2) \tau_{n}+n \chi_{n} ; \\
& f_{2}=n \tau_{n}, \quad g_{2}=\chi_{n}+n \chi_{n n} ; \\
& f_{3}=(1 / 4) \tau-n \tau_{n}-(5 / 4) w n \chi_{n}+\left(-n+(\alpha / 4) w^{2}\right) n \tau_{n n}-w n^{2} \chi_{n n}, \\
& g_{3}=(3 / 4) v \tau_{n}-\left(2 n-(\alpha / 4) w^{2}\right) \chi_{n}+\alpha w n \tau_{n n}+\left(-n+(\alpha / 4) w^{2}\right) n \chi_{n n} .
\end{aligned}
$$

The first operator with the coordinates $f_{1}$ and $g_{1}$, which are linear in the first derivatives, is equivalent to the point symmetry operator; the other two operators are the Lie-Bäcklund 
symmetry operators of the second order. Restricting the Lie-Bäcklund group found for the RG manifold consists in verifying the FS condition

$$
f=0, \quad g=0,
$$

which must be satisfied on the solution of the boundary-value problem and be consistent with boundary conditions (30). The form of the coordinates $f$ and $g$ implies that these conditions, written in terms of the boundary function $H(n)$, must satisfy linear PDEs with variable coefficients. In particular, for the case $N(x)=\cosh ^{-2}(x)$, the FS condition is fulfilled for the combinations $f=f_{1}+2\left(f_{2}+f_{3}\right)$ and $g=g_{1}+2\left(g_{2}+g_{3}\right)$, and the desired Lie-Bäcklund RGS operator is

$$
\begin{aligned}
& R_{7}=\left(2 n(1-n) \tau_{n n}-n \tau_{n}-2 n w\left(\chi_{n}+n \chi_{n n}\right)+\frac{\alpha}{2} n w^{2} \tau_{n}\right) \partial_{\tau} \\
& +\left(2 n(1-n) \chi_{n n}+(2-3 n) \chi_{n}+\alpha w\left(2 n \tau_{n n}+\tau_{n}+\frac{w}{2}\left(n \chi_{n n}+\chi_{n}\right)\right)\right) \partial_{\chi} .
\end{aligned}
$$

Condition (34) for the operator $R_{7}$ results in a second-order PDE, which distinguishes it qualitatively from the first-order equations arising in the FS conditions considered previously. Namely, the finite FS transformations for the latter can be found in a closed form as the solutions of the corresponding Lie equations expressing the transformations of dependent and independent variables and parameters. The solutions of the Lie equations for the RGS with Lie-Bäcklund operator (35) result in finite transformations written as formal power series. However, this does not mean that FS conditions have no practical importance in this case; on the contrary, the differential formulation of the FS conditions permits considering relations (34) as additional differential constraints, which must be taken into account when finding solutions of the boundary-value problem. The solution of the initial equations with the differential constraints taken into account leads to the solution of the boundary-value problem 20, 21, 22] that is invariant w.r.t. RGS group (35).

To conclude this section, we note that the FS conditions written as vanishing conditions for coordinates of a Lie-Bäcklund canonical RG operator, being considered as a system of differential constraints, play the role of embedding equations. Imposing the FS conditions, we come to the problem of finding the solution that is invariant w.r.t. the found RGS operator. A more informative example is provided by using the FS conditions to find point RGS operators as the group admitted by the initial equations and FS relations [15]. There, RGS operators containing higher-order derivatives w.r.t. parameters entering the equations can be discussed in principle.

\section{The FS and RGS for systems with small parame- ters}

We now consider the FS property for systems described by DEs with small parameters. If a system contains a small parameter $\alpha$, we can start the RGS construction by considering 
the simplified $(\alpha=0)$ model, which admits a wider symmetry group in comparison with the case $\alpha \neq 0$. When we take the contributions from small $\alpha$ into account, this symmetry is inherited by the initial system of equations, which results in the appearance of additional terms, corrections in powers of $\alpha$, in the operator coordinates. Restricting the obtained symmetry group to an exact or approximate solution of the boundary-value problem, we obtain the desired RGS, which can be also represented as operators whose coordinates are infinite series in powers of small parameters. For boundary data of a special form, these series terminate, which results in an exact RGS without restrictions on values of the relevant parameters. For boundary data of a general type, the procedure of truncating the infinite series to a finite number of terms leads to an approximate RGS at small parameter values.

\subsection{FS conditions in nonlinear plasma theory}

We now consider the boundary-value problem for the equations of the nonlinear interaction of laser radiation with a plasma [23]. Such an interaction for a $p$-polarized electromagnetic wave of frequency $\omega$, for which only the $z$ component of the magnetic field is nonzero and which propagates from the vacuum toward an inhomogeneous plasma, is described by a system (we do not present it here) of nonlinear nonstationary equations for six scalar functions: two components of the electron velocity $v_{x}$ and $v_{y}$, the electron density $n$, two electric field components $E_{x}$ and $E_{y}$, and the $z$ component $B_{z}$ of the magnetic induction; these functions depend on the time $t$ and the two coordinates $x$ and $y$.

The nonlinearity of these equations is essential in a small space domain near the plasma resonance (at $\omega_{L}^{2} \approx \omega^{2}$ ) where the presence of natural small parameters (such as the smooth inhomogeneity of the ion density $N(x)$ along the $x$ axis and the small incidence angles $\vartheta$ of laser beams at the plasma) implies the appearance of a hierarchy of components of the $p$-polarized light wave at the critical plasma point. When constructing the inherited point RGS, this allows reducing the total system of six initial equations to a simpler system of two one-dimensional nonlinear PDEs for the components of the electron velocity $v$ and the electric field $E$ along the density gradient vector [24, 23],

$$
v_{t}+a v v_{x}-E=0 ; \quad E_{t}+a v E_{x}+\omega_{L}^{2} v=0, \quad \omega_{L}^{2} \equiv \frac{4 \pi\left|e e_{i}\right| N}{m} .
$$

Here, the functions $v$ and $E$ are expressed in units of the dimensionless nonlinearity parameter $a$, which is proportional to the value of the magnetic induction $B$ at the critical point at the laser frequency; the coordinate $y$ enters only in combination with time, $t \rightarrow t-(\vartheta y / c)$.

The system of six initial equations admits only a finite group of point transformations, namely, the group of translations along the $t$ and $y$ axes for arbitrary $N(x)$. At a constant ion density, $N=$ const, the additional group of $x$-axis translations and the group of simultaneous rotations in three planes, which are determined by the coordinates $\{x, y\}$ and the corresponding $x$ and $y$ components of the electron velocity and of the electric 
field, arise. In contrast to the initial equations, system (36) admits an infinite group of point transformations with the operator containing the three terms

$$
\begin{gathered}
X_{1}=\mu_{1} Y ; \quad X_{2}=\mu_{2} \partial_{x}+\frac{1}{a} Y\left(\mu_{2}\right) \partial_{v}+\frac{1}{a} Y^{2}\left(\mu_{2}\right) \partial_{E} ; \\
X_{3}=\frac{1}{a} \mu_{3}\left(a \partial_{a}-v \partial_{v}-E \partial_{E}\right) ; \quad Y=\partial_{t}+a v \partial_{x}+E \partial_{v}-\omega_{L}^{2} v \partial_{E},
\end{gathered}
$$

each of which contains an arbitrary function $\mu_{i}$ of independent and dependent variables and of the parameter $a$ while the differential constraints

$$
Y^{3}\left(\mu_{2}\right)+Y\left(\omega_{L}^{2} \mu_{2}\right)=0 ; \quad Y\left(\mu_{3}\right)=0 .
$$

are imposed on $\mu_{2}$ and $\mu_{3}$.

To obtain the RGS, point transformation group (37) must be restricted on a solution of the boundary-value problem that is approximate over the powers of the parameter $a$; this solution is such that the leading approximation for the functions $v$ and $E$ is determined by a solution of the linearized system of initial equations with the corresponding boundary conditions (the propagation of the electromagnetic wave from the vacuum toward the plasma) and with the given shape of the density $N(x)$ in the plasma resonance domain taken into account; the corrections proportional to $a$ appear when linearizing system (36). Verifying the FS conditions for group (37), we find that $\mu_{1}=0, \mu_{2}=-E / \omega^{2}$, and $\mu_{3}=1$ for this particular solution, which gives the desired RGS operator

$$
R_{8}=X_{2}+X_{3}=-\frac{E}{\omega^{2}} \partial_{x}+\partial_{a}
$$

The condition of invariance of the solution of the boundary-value problem w.r.t. the RG operator $R_{8}$ (the FS condition) is described by the system of first-order PDEs

$$
v_{a}-\frac{E}{\omega^{2}} v_{x}=0, \quad E_{a}-\frac{E}{\omega^{2}} E_{x}=0 .
$$

The solution of the characteristic equations for this system (the system of Lie equations for the operator $R_{8}$ ) reconstructs the $\mathrm{PT}$ in the parameter $a$ up to the exact solution of the boundary-value problem [23,

$$
\begin{gathered}
E=-\frac{(\omega L)^{2}}{\Delta}\left(q_{1} \sin \omega t+q_{2} \cos \omega t\right), \quad v=-\frac{\omega L^{2}}{\Delta}\left(q_{1} \cos \omega t-q_{2} \sin \omega t\right), \\
x=\mu+\varepsilon\left(q_{1} \sin \omega t+q_{2} \cos \omega t\right), \quad \varepsilon=a L^{2} / \Delta^{2},
\end{gathered}
$$

which describes the nonlinear structure of the electric field in the plasma resonance domain. The dimensionless quantities $x$ and $\mu$ are normalized to the resonance width $\Delta$, and $L$ is the characteristic inhomogeneity scale of the plasma ion density. The choice of the 
form of the functions $q_{1}$ and $q_{2}$ in relations (40) is determined by the actual dependence on the $x$ coordinate of the electric field, which is a solution of the initial total system of equations under the corresponding boundary conditions and with the given shape of the density in the plasma resonance domain.

For example, for a cold electron plasma with a linear density function, the functions $q_{1}$ and $q_{2}$ are

$$
q_{1}=\left(1+\mu^{2}\right)^{-1}, \quad q_{2}=\mu\left(1+\mu^{2}\right)^{-1},
$$

where the width $\Delta=(\nu / \omega) L$ is determined by the frequency of plasma particle collisions.

For a hot plasma in which the heat motion of electrons is essential, linearizing the initial system of equations, we find that in the case where the density function is linear in the plasma resonance domain, the electric field distribution is expressed through the Airy-Fock functions. The corresponding nonlinear structures of the electric field and of the density and velocity of electrons, which appear from using RG operator (38) to continue the linear relations with the first nontrivial heat correction (for $a \rightarrow 0$ ) to finite values of $a$, are determined, as before, by expressions (40) in which the resonance width $\Delta$ now depends on the electron temperature, $\Delta=\left(3 V_{T}^{2} L / \omega^{2}\right)^{1 / 3}$, and the functions $q_{i}$ are

$$
q_{1}=\int_{0}^{\infty} d \xi \cos \left(\mu \xi+\xi^{3} / 3\right), \quad q_{2}=\int_{0}^{\infty} d \xi \sin \left(\mu \xi+\xi^{3} / 3\right) .
$$

A feature of the formulas for $v$ and $E$ in (40) with the functions $q_{1}$ and $q_{2}$ from (42) is that they give the exact (at $\omega_{L}^{2}=\omega^{2}$ ) solution of Eqs. (36), in which the electron pressure is neglected but the nonzero electron temperature is nevertheless taken into account.

These formulas as well as their physical consequences were analyzed in detail in [23, 25]. Here, we only note the value of the results obtained: the FS condition for a simple mathematical model leads to proper results even in the leading order in which small corrections to RGSs are neglected (although a modification of RG operator (38) taking these corrections into account is not complicated; the corresponding expression for corrections in the density gradient was given in [23]). The big freedom in choosing the functions $q_{1}$ and $q_{2}$ in Eqs. (40) permits analyzing the nonlinear structure of the electric field in the plasma resonance domain in both cold and hot plasmas uniformly.

We note that RG operator (38) is qualitatively analogous to Bogoliubov RG operator (4). As in the standard QFT RG case, operator (38) determines the translation transformation along a solution characteristic (the parameter $a$ ) as well as more complicated functional transformations of the coordinate $x$. The FS property of the physical system under investigation is the invariance of the desired functions, the field $E$ and electron velocity $v$, w.r.t. these RG transformations. Then, the nonlinearity parameter $a$, which enters the RG transformation, is not assumed to be small in contrast to the terms dropped when obtaining the operator $R_{8}$ of the "approximate" RGS; the smallness of these terms was crucial for passing from the initial system of equations to Eqs. (36). Therefore, the RGS can be used to extend the solution obtained in the form of a power 
series in the parameter $a$ to the exact, essentially nonlinear solution, Eqs. (40). Then, as in the QFT case, the obtained RG operator corresponds to the exact group transformation w.r.t. the parameter $a$ for a solution of Eqs. (36). At the same time, this RG operator is approximate because it was obtained by neglecting parameters other than $a$ and admits a subsequent improvement w.r.t. those parameters.

\subsection{The FS in nonlinear wave optics}

In many cases, we cannot neglect all but leading (zero-order in small parameters) contributions to an RGS. Therefore, we consider a variant of the RGS and FS conditions that explicitly takes contributions of small parameters into account in the RG operator; such a modification appears when analyzing the boundary-value problem, which generalizes (28) and (29),

$$
\begin{gathered}
v_{t}+v v_{x}-\alpha n_{x}-\beta \partial_{x}\left(\left(x^{1-\nu} / \sqrt{n}\right) \partial_{x}\left(x^{\nu-1} \partial_{x}(\sqrt{n})\right)\right)=0, \\
n_{t}+n v_{x}+v n_{x}+(\nu-1) \frac{n v}{x}=0 .
\end{gathered}
$$

Here, $\alpha$ is the nonlinear refraction parameter as previously, $\beta$ is the parameter that defines the diffraction effects, and the respective values $\nu=1$ and $\nu=2$ correspond to flat and cylindrical geometries of the beam. Boundary conditions for Eqs. (43) determine the curvature of the wave front of the beam and its intensity distribution over the transverse coordinate $x$,

$$
v(0, x)=V(x)=-x / T, \quad n(0, x)=N(x) .
$$

\subsubsection{Flat geometry}

We now set the geometry to be flat $(\nu=1)$, neglect the diffraction $(\beta=0)$, and consider the simplest case where the wave beam on the medium boundary (i.e., at $t=0$ ) has a flat wave front. Then, Eqs. (43) become system (30). We write the RGS operators for boundary-value problem (30), (31) in canonical form (32). We represent the coordinates $f$ and $g$ as power series,

$$
f=\sum_{i=0}^{\infty} \alpha^{i} f^{i} ; \quad g=\sum_{i=0}^{\infty} \alpha^{i} g^{i} .
$$

The method for calculating the coefficients $f^{i}$ and $g^{i}$ and the resulting system of recursive relations for these coefficients was derived in [26, 27]. There, each coefficient was determined up to an arbitrary function of $n, \chi_{(s)}$, and of the combination $\tau_{(s)}-w\left(s \chi_{(s)}+n \chi_{(s+1)}\right)$ where $(s)$ denotes the $s$ th-order derivative in $n$. This arbitrariness can be removed by restricting the group on the desired solution of the boundary-value problem, i.e., by imposing FS condition (34), which becomes a differential or algebraic constraint that is compatible with boundary condition (31) at $\tau=0$. Truncating series (45), which is possible at small values of $\alpha$, we obtain an approximate symmetry; the contributions $f^{0}$ 
and $g^{0}$, which do not depend on the parameter $\alpha$, are then determined by the system of equations

$$
\tau_{w}-n \chi_{n}=0, \quad \chi_{w}=0 .
$$

which is simpler than initial system (30). In contrast to system (30), which admits only a finite group of Lie-Bäcklund transformations of a given order, system (46) admits an infinite symmetry group because the coordinates $f^{0}$ and $g^{0}$ can be arbitrary functions of their arguments. At small $\alpha$, the symmetry of Eqs. (46) is inherited by initial system (30) up to an arbitrary given order in $\alpha$. Restricting the obtained approximate group on the solution of the boundary-value problem, we obtain the desired RGS.

We now turn to RGS operators with coordinates in the form of the binomials

$$
f=f^{0}+\alpha f^{1}, \quad g=g^{0}+\alpha g^{1} .
$$

These operators depend on the functions $f^{0}$ and $g^{0}$, which, in turn, can be (nonuniquely) determined by the function $N(x)$ of the transverse intensity distribution at the boundary of the nonlinear medium.

For the "soliton" beam intensity distribution function $N(x)=\cosh ^{-2} x$, we can obtain two sets of formulas for the coefficients resulting from different expressions for $f^{0}$ and $g^{0}[26]$ :

$$
\begin{aligned}
& f^{0}=2 n(1-n) \tau_{n n}-n \tau_{n}-2 n w\left(\chi_{n}+n \chi_{n n}\right), f^{1}=\frac{1}{2} n w^{2} \tau_{n n}, \\
& g^{0}=2 n(1-n) \chi_{n n}+(2-3 n) \chi_{n}, g^{1}=w\left(2 n \tau_{n n}+\tau_{n}\right)+\frac{w^{2}}{2}\left(n \chi_{n n}+\chi_{n}\right) . \\
& \text { b) } f^{0}=1+2 n \chi_{n} \tanh \chi, f^{1}=\left(\frac{\tau^{2}}{n}-2 \tau \tau_{n}+2 \tau^{2} \tanh \chi\right) \cosh ^{-2} \chi, \\
& g^{0}=0, g^{1}=-2 \tau \chi_{n} \cosh ^{-2} \chi-2 \tau_{n} \tanh \chi .
\end{aligned}
$$

Calculating contributions of higher orders [26, 28, 20], we find that the functions $f^{i}$ and $g^{i}$ vanish for $i \geq 2$ in case a and formulas (48) and (45) describe an exact RGS; the comparison with coordinates (35) demonstrates that the functions $f^{1,2}$ and $g^{1,2}$ are the coefficients of the coordinate expansions in powers of $\alpha$. In case $b$, series (45) do not terminate, and formulas (49) and (45) pertain to an approximate RGS.

For the wave beam with a Gaussian initial intensity shape, $N(x)=\exp \left(-x^{2}\right)$, we have two sets of the $f$ and $g$ operators of an approximate RGS:

$$
\begin{aligned}
& \text { a) } f^{0}=1+2 n \chi \chi_{n}, \quad g^{0}=0, \quad f^{1}=-2 \tau \tau_{n}+\frac{\tau^{2}}{n}, \quad g^{1}=-2\left(\tau \chi_{n}+\chi \tau_{n}\right), \\
& \text { b) } f^{0}=2 n\left(\tau \chi_{n}+\tau_{n} \chi\right), \quad g^{0}=1+2 n \chi \chi_{n}, \quad f^{1}=2 \chi \tau_{\alpha}, \quad g^{1}=2\left(\chi \chi_{\alpha}-\tau \tau_{n}\right) .
\end{aligned}
$$


Formulas (48)-(51) demonstrate the main advantage of the approximate RGS method, which permits analyzing boundary-value problems with arbitrary boundary data, which are expressed through differential or algebraic expressions for the functions $f^{0}$ and $g^{0}$. The example of operator (48) shows that in some cases the approximate RGS method can result in an exact RGS whose presence must be established using the tools in the previous section. Because expressions (48) for the coordinates $f^{i}$ and $g^{i}$ contain secondorder derivatives, the corresponding operator $R$ is the RGS Lie-Bäcklund operator of the second order, while operators (49)-(51) are equivalent to point symmetry operators. Meanwhile, because operator (51) contains the first derivative in the parameter $\alpha$, the FS transformations also involve the nonlinear refraction parameter [26]. (See [26, 22] for a detailed analysis of formulas (48)-(51) and the physical consequences of their substitution in FS conditions (34).)

\subsubsection{Cylindrical case}

Analogously to the previous case, approximate RGSs for the case $\beta \neq 0$ can be constructed using FS conditions [27]. An example is the RGS operator for the cylindrical $(\nu=2)$ wave beam,

$$
\begin{aligned}
& R_{9}=\left[\left(1-\frac{t}{T}\right)^{2}+t^{2} S_{\chi \chi}\right] \partial_{t}+\left[-\frac{x}{T}\left(1-\frac{t}{T}\right)+t S_{\chi}+v t^{2} S_{\chi \chi}\right] \partial_{x} \\
& +\left[\frac{x}{T^{2}}+\frac{v}{T}\left(1-\frac{t}{T}\right)+S_{\chi}\right] \partial_{v}+\left[\frac{2 n}{T}\left(1-\frac{t}{T}\right)-n t\left(1+\frac{v t}{x}\right) S_{\chi \chi}-\frac{n t}{x} S_{\chi}\right] \partial_{n} .
\end{aligned}
$$

where the function $S$ depends on the variable $\chi=x-v t$,

$$
S(\chi)=\alpha N(\chi)+\frac{\beta}{\chi \sqrt{N(\chi)}} \partial_{\chi}\left(\chi \partial_{\chi}(\sqrt{N(\chi)})\right),
$$

contains two small parameters $\alpha$ and $\beta$, and is determined by the initial beam intensity distribution function $N$ at the boundary of the nonlinear medium. Then, as in the case $\beta=0$, there exist such functions $N$ for which operator (52) corresponds to an exact, rather than approximate, RGS, i.e., the RGS that is applicable at arbitrary, not necessarily small, values of the parameters $\alpha$ and $\beta$ [27]. The FS transformations appear from the solution of the characteristic equations for the first-order PDE that is conjugate to (52) and permit continuing the PT solutions, which are determined only in a small vicinity of the nonlinear medium boundary, to a domain where essentially nonlinear effects prevail [27].

To conclude this section, we again note that we can write the FS conditions in many different ways, which implies different approaches to their subsequent use in problems with a small parameter. The use of approximate symmetries results in some peculiarities in constructing the RGSs and in using the FS conditions. 
First, as discussed in the previous section, we apply the FS conditions in the form of a system of higher-order DEs and analyze them together with the initial equations. In contrast to finite FS transformations, which are infinite formal series (see [18], Vol. 3, Chap. 1), the differential formulation of the FS conditions is suitable for constructing solutions of a boundary-value problem.

Second, the FS conditions expressed as approximate symmetries are additional differential constraints, which together with the initial equations determine the RG manifold. In turn, such a manifold can be used to construct approximate point RGSs.

Third, the FS conditions are important at all stages of constructing approximate RGSs already starting with setting the RGS operator coordinates for an unperturbed solution. New prospects are provided by the possibility of constructing RGSs based on approximate symmetries for problems with arbitrary boundary conditions.

Fourth, we introduce parameters that are used to construct approximate RGSs in the set of variables of the FS transformations.

\section{Conclusion}

We now formulate how the FS notion has been transformed in the last decade. This notion first appeared as pertaining to the group transformations developed by Lie in 29], which was used to construct the QFT (Bogoliubov) RG [4, 5]. The latter concept was based on the one-parameter Lie group of local transformations, the class the Bogoliubov RG belongs to. The FS notion unified various RGs and exhibited their intrinsic group structure.

The progress in applying mathematical methods to the investigation of symmetries of differential and integral-differential equations, which resulted in modern group analysis (see, e.g., 110, 18, 30, 31] and references therein), has led to the transformation of the FS notion.

First, this resulted in constructing the special class of symmetries related to the RG transformations, namely, the RGSs. Such a method (see Sec. 2) regularizes the procedure for finding RG transformations, at least for systems that are described by differential and integral-differential equations. Using the infinitesimal approach, we can formulate the RG invariance conditions through the RGS operators and can therefore use all the powerful tools of modern group analysis to construct these conditions. As a result, the RGS notion now includes not only point symmetries (Sec. 3) but also Lie-Bäcklund symmetries (higher, or generalized, symmetries) (Secs. 4 and 5), approximate symmetries (Sec. 5), etc. The list of examples, which is far from complete, presented in this paper includes the most advanced results in constructing the RGSs; furthermore, our approach is equally applicable to the cases of nonlocal RGSs and to RGSs of integral-differential equations if, for example, we apply the method described in [32]. Therefore, modern group analysis is as important for developing the FS notion as was the classical Lie group analysis for establishing the Bogoliubov RG. 
The FS conditions are formulated as conditions of invariance w.r.t. transformations determined by RG operators. This becomes important when the formulation of finite FS transformations is doubtful, e.g., for the Lie-Bäcklund RGSs (see Secs. 4 and 5). Being universal, this formulation is useful not only at the stage of finding the solutions but also when constructing RGS operators. Thus, the FS conditions and the procedure for their verification became important components of the RGS construction. The conditions themselves can be algebraic as well as differential relations containing derivatives of higher (not necessarily first, see Sec. 4.2) order.

A feature of the new way of constructing RGSs is the regular procedure for constructing Lie algebras of finite dimension. Numerous examples of such algebras are given in 9, 19., although they have not yet found wide practical applications (see Sec. 3). Such Lie algebras correspond to multiparameter groups. The RGS apparatus results in a set of group operators constituting a Lie algebra. Such groups are customarily used by researchers into symmetries of equations arising in contemporary theoretical physics.p However, the "renormalization group" in both the QFT (Bogoliubov) and the Wilson approaches was always assumed to be a one-parameter group. Rare attempts to consider two-parameter constructions have always (to the best of our knowledge [33]) led to direct products of two one-parameter RGs.

The RG technique was customarily used in problems with singularities to improve the PT and to give a correct description of the solution behavior in the vicinity of a singularity; these properties are intrinsic in our approach as well. Namely, using the FS conditions formulated in Secs. 4 and 5 on the base of the Lie-Bäcklund RGS, we were able not only to describe the structure of the known singular solutions but also to find new ones (see [22, 26, 27]). We stress that the RGS approach to these problems results in a structure of two-dimensional singularities that differs from the structure of singularities appearing within the Bogoliubov RG setting.

\section{Acknowledgments}

This work was supported in part by the Russian Foundation for Basic Research (Grant Nos. 96-15-96030 and 99-01-00232) and INTAS (Grant No. 96-0842).

\section{References}

[1] D. V. Shirkov, Sov. Phys. Doklady 27 (1982) 197.

[2] D. V. Shirkov, Theor. Math. Phys. 60 (1984) 778.

[3] D. V. Shirkov, Intern. J. Mod. Physics A3 (1988) 1321.

\footnotetext{
${ }^{3}$ For instance, the basis of the Poincaré group transformation algebra consists of 10 generators.
} 
[4] E. C. G. Stückelberg and A. Petermann, Helv. Phys. Acta 26 (1953) 499.

[5] N. N. Bogoliubov and D. V. Shirkov, Dokl. Akad. Nauk SSSR 103 (1955) 203; 391 in Russian; see also N. N. Bogoliubov and D. V. Shirkov, Sov. Phys. JETP 3 (1956) 57; N. N. Bogoliubov, D. V. Shirkov, Nuovo Cim. 3 (1956) 845.

[6] K. Wilson, Phys. Rev. B4 (1971) 3184.

[7] N. N. Bogoliubov and D. V. Shirkov, Introduction to the Theory of Quantum Fields, New York, Wiley, 1980.

[8] V. Z. Blank, V. L. Bonch-Bruevich, and D. V. Shirkov, Sov. Phys. JETF 6 (1958) 204.

[9] V. F. Kovalev, V. V. Pustovalov, and D. V. Shirkov, J. Math. Phys. 39 (1998) 1170.

[10] L. V. Ovsiannikov, Group Analysis of Differential Equations, New York, Acad. Press, 1982.

[11] L.-Y. Chen, N. Goldenfeld, and Y. Oono, Phys. Rev. E 54 (1996) 376.

[12] J. Bricmont, A. Kupiainen and G. Lin, Comm. Pure Appl. Math. 47 (1994) 893.

[13] T. Kunihiro, Progr.Theor.Phys. 94 (1995) 503.

[14] V. F. Kovalev, S. V. Krivenko, V. V. Pustovalov, The Renormalization group method based on group analysis, in Renormalization group-91, Eds D.V. Shirkov \& V.B.Priezhzev (World Scientific, Singapore, 1992), 300-314.

[15] V. F. Kovalev, V. V. Pustovalov, and D. V. Shirkov, Group analysis and renormgroup [in Russian], Communications of JINR, P5-95-447, Dubna (1995).

[16] R. M. Corless et al., Adv. Comp. Math. 5 (1996) 329.

[17] D. V. Shirkov and I. L. Solovtsov, Theor. Math. Phys. 120 (1999) 1220.

[18] CRC Handbook of Lie Group Analysis of Differential Equations, edited by N.H. Ibragimov, 3 vols., CRC Press, Boca Raton, Florida, USA, 1994-1996.

[19] V. F. Kovalev, V. V. Pustovalov, Lie Group and their Applications 1 (1994) 104.

[20] V. F. Kovalev, V. V. Pustovalov, Mathl. Comput. Modelling 25 (1997) 165.

[21] V. F. Kovalev, J. Nonlin. Math. Phys. 3 (1996) 351.

[22] V. F. Kovalev, D. V. Shirkov, J. Nonlinear Opt. Phys. \& Materials 6 (1997) 443.

[23] V. F. Kovalev and V. V. Pustovalov, Theor. Math. Phys. 81 (1989) 1060. 
[24] V. F. Kovalev and V. V. Pustovalov, Functional self-similarity in nonlinear plasma theory, in Soviet Physics - Lebedev Institute Reports, No. 3, 1989, 54.

[25] V. F. Kovalev and V. V. Pustovalov, Sov. J. Quantum Electron. 18 (1988) 463; 19 (1989) 1454. Sov. J. Plasma Phys. 15 (1989) 27; 15 (1989) 327.

[26] V. F. Kovalev, Theor. Math. Phys. 111 (1997) 686.

[27] V. F. Kovalev, Theor. Math. Phys. 119 (1999) 719.

[28] V. F. Kovalev, V. V. Pustovalov, and S. I. Senashov, Diff. Equat. 29 (1993) 1521.

[29] S. Lie, Gesammelte Abhandlungen, 6 vols. Leipzig, B.G.Teubner, 1922-1927.

[30] Peter J. Olver, Applications of Lie groups to differential equations, New York, Springer-Verlag, 1986.

[31] A. M. Vinogradov and I. S. Krasil'shchik, eds. Symmetries and Conservation Laws of Equations of Mathematical Physics, Moscow, Faktorial, 1997.

[32] V. F. Kovalev, S. V. Krivenko, and V. V. Pustovalov, Diff. Equat. 29 (1993) 1568; 29 (1993) 1712.

[33] C. Stephens, Int. J. Mod. Phys. 12 (1998) 1379; Mod. Phys. Lett. A9 (1994) 309. 


\section{Contents}

\begin{tabular}{llr}
\hline 1 & Introduction & 1
\end{tabular}

2 The QFT RG and the FS condition 3

2.1 Simple Bogoliubov RG transformations . . . . . . . . . . . . . . . . . . . . 3

2.2 Self-similarity and the FS . . . . . . . . . . . . . . . . . . . . . . . . . . . . . . . .

2.3 Constructing the RGS $\ldots \ldots \ldots \ldots \ldots \ldots$

\begin{tabular}{|llllll}
\hline 3 & The FS analysis of systems that are close to quantum field systems & 7
\end{tabular}

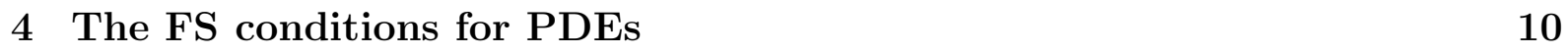

4.1 Boundary-value problem for the Burgers equation . . . . . . . . . . . . . . 11

4.2 The boundary-value problem for nonlinear optic equations . . . . . . . . . . 12

5 The FS and RGS for systems with small parameters 14

5.1 FS conditions in nonlinear plasma theory . . . . . . . . . . . . . . . . . 15

5.2 The FS in nonlinear wave optics . . . . . . . . . . . . . . . . . . . . . . . . 18

5.2 .1 Flat geometry . . . . . . . . . . . . . . . . . . . . . 18

5.2 .2 Cylindrical case . . . . . . . . . . . . . . . . . 20

\begin{tabular}{lll}
\hline 6 Conclusion & 21 \\
\hline
\end{tabular}

\begin{tabular}{lll}
\hline 7 & Acknowledgments & 22
\end{tabular}

\begin{tabular}{ll}
\hline References & 22
\end{tabular} 